\section{Cahiers de Narratologie}

Analyse et théorie narratives

22 | 2012

Voix off et narration cinématographique. Second volet

\title{
Maurice Blanchot : une phénoménologie du récit
}

\section{Maxime Decout}

\section{OpenEdition}

Journals

Édition électronique

URL : http://journals.openedition.org/narratologie/6572

DOI : 10.4000/narratologie.6572

ISSN : $1765-307 X$

Éditeur

LIRCES

\section{Référence électronique}

Maxime Decout, « Maurice Blanchot: une phénoménologie du récit », Cahiers de Narratologie [En ligne], 22 | 2012, mis en ligne le 20 juillet 2012, consulté le 19 avril 2019. URL : http:// journals.openedition.org/narratologie/6572; DOI : 10.4000/narratologie.6572

Ce document a été généré automatiquement le 19 avril 2019

Article L.111-1 du Code de la propriété intellectuelle. 


\title{
Maurice Blanchot : une phénoménologie du récit
}

\author{
Maxime Decout
}

1 Maurice Blanchot a manifestement beaucoup plus compté comme théoricien que comme romancier, et ce alors même que sa poétique, dès Thomas l'obscur en 1932, s'affiche comme essentiellement novatrice. L'écrivain n'a cessé de pousser dans ses implications les plus ultimes les nouvelles versions et subversions qu'il faisait subir au récit, et dont le point d'aboutissement semble être L'Attente l'oubli. Cet ensemble de distorsions de l'espace narratif est l'expression de la recherche incertaine et difficile d'une nouvelle appréhension du réel, dans le sillage de la pensée philosophique de son temps, telle qu'on la trouve dans ses essais, chez Derrida ou chez Bataille ${ }^{1}$. Son écriture met en scène une sorte de déconstruction des couples notionnels binaires de la pensée traditionnelle, ouvrant à des logiques paradoxales loin des catégories usuelles. L'œuvre de Blanchot invite ainsi à traquer l'impensé qui se love au creux de nos systèmes de pensée institués. Loin du dogmatisme péremptoire où se fourvoient les rêves de totalité de la littérature, ses fictions soulignent d'un trait fuyant les zones fragiles où l'incertain se doit d'être mesuré plus que débouté.

2 Et c'est avec Derrida, tel qu'il lit Blanchot, qu'il convient peut-être de cerner le questionnement central proposé par ses textes: «Qu'est-ce qu'un récit? Cette chose qu'on appelle récit? A-t-elle lieu ? Où et quand? Quel serait l'avoir-lieu ou l'événement d'un récit ${ }^{2}$ ? C'est assurément cet espace problématique qu'invitent à questionner les œuvres de Blanchot et, tout particulièrement, L'Attente l'oubli où cette interrogation est portée à son incandescence. Car ses fictions interrogent les liens entre le récit et l'événement, questionnent le récit comme pensée de l'événement, et même plus, inquiètent l'événement du récit en lui-même. En effet, les conditions du récit blanchotien sont avant tout celles d'un évidemment du réel, auxquelles répond un phénomène identiquement relayé dans la pratique narrative. La pensée, la parole, l'être, en tant que tels, sont les objets d'une quête. Réalités intangibles et secrètes, ils commandent une sorte de mystique littéraire qui doit puiser dans une obscurité concertée afin de les 
approcher. Tout le problème de la littérature chez Blanchot réside ainsi dans la découverte des moyens de cette quête.

Au départ de cette croisade, se tient l'évidence que le monde est une entité entamée par le sens absent de la Nuit et du Dehors ${ }^{3}$, qui échappe par avance à toute saisie et ne se laisse entrevoir que dans une phénoménologie problématique. En effet, Marlène Zarader affirmait bien que l'œuvre de Blanchot «n'a de sens (et de force) que replacée sur un sol phénoménologique dont, en même temps, elle veut s'extraire ${ }^{4}$ ", en effondrant notamment la place du sujet et de la conscience. Si les sens multiples à même d'approcher le réel, que les textes convoquent comme viatiques incertains, et dont les plus essentiels sont assurément la vue et l'ouie, sont vacillants, l'efficacité de la pensée et de la parole n'en est pas moins douteuse. Ce tremblement dans les moyens de saisie du monde repose sur le fait que l'univers, dans les récits de Blanchot, est essentiellement un apparaître, et la parole, l'être et la pensée en sont les soutènements les plus authentiques mais aussi les plus fuyants. Comment en ce cas saisir un réel constitué avant tout des entités à même de le percevoir que sont la parole et la pensée ? Utiliser comme moyen d'appréhension la matière même de ce que l'on cherche à approcher semble confiner l'écrivain dans une tautologie inexorable où le dire et le dit se constitueraient comme la tragique confirmation de ce qui est, et non comme son exploration. La littérature se ferait ainsi le témoin du monde et du Dehors, et non leur rationalisation. Confronté à cette impuissance du langage et de la pensée, le romanesque de Blanchot doit redéfinir les moyens qui lui sont propres. L'objectif visé serait ainsi proche de celui de la réflexion philosophique de Blanchot qui cherche à « ordonner son dire (...) à cette part obscure de l'expérience, celle qui se déploie à l'écart de tout possible et qui défie, en conséquence, toute nomination ${ }^{5}$ ». Et pourtant, «cela ne signifie pas qu'une parole ne puisse lui être consacrée; mais elle devra se déployer, au même titre que son objet, dans un espace impossible ${ }^{6} »$. Cet espace, outre celui de la parole réflexive à laquelle Marlène Zarader fait allusion, est aussi celui du récit. Refusant de la sorte l'illusion d'une consistance du réel ou de l'événement, c'est leur forme et leur apparence telles qu'elles surgissent que le récit doit viser. Ainsi conçue, la pratique narrative tend à s'apparenter à une étrange phénoménologie romanesque où les substances et les contenus s'évident au profit des formes qui les portent.

\section{Description d'un instant}

Dans l'espace incertain du récit chez Blanchot, les prérogatives du réel sont avant tout celles d'une temporalité réglée sur une norme unique: l'instant. Les titres indiquent clairement ce chemin désigné à notre imaginaire habitué à l'épaisseur de la durée : L'Arrêt de mort, Au Moment voulu, L'Instant de ma mort. Entité flottante, qui glisse entre les êtres aussi bien qu'entre les mots qui essayent de l'appréhender, l'instant se conçoit comme une fulgurance. Les textes cherchent ainsi à recueillir le présent et la présence, tels qu'ils se donnent dans leur dérobade même. Pourtant, cet éclair de l'instant, à être mis en mots, s'étend au-delà de lui-même. La succession des mots, l'ordre syntaxique, les nécessités, quoique toujours contestées, de la mise en récit, imposent inévitablement une durée à ce qui n'en a pas. C'est ainsi que Bergson soulignait, quant à la sensation, la problématique utilisation du langage dans le rendu de ce qui, par nature, lui échappe, car les mots, « inventés pour témoigner que la sensation est instable, (...) imposeraient leur propre stabilité ${ }^{\prime}$ à la pensée. Il en irait de même pour la fixation de l'instant. 
Dans l'ordre du dit comme de l'écrit, l'instantanéité reste donc une chimère. Aussi, l'impossibilité de la répétition est-elle partout soulignée: «Elle avait voulu le répéter, mais ce fut en vain; par la suite, et malgré tous ses efforts pour le lui faire redire, elle ne put jamais retrouver l'expression dont elle s'était servie ${ }^{8}$ ». Plus précisément ce n'est pas la répétition en tant que telle qui s'avère impossible mais bien la re-présentation, l'actualisation à nouveau d'un instant premier dérobé : «Le redire, c'est facile, mais le dire encore une première fois?» $(A O, 101)$. Le dit s'inscrit dans une unicité et une instantanéité qui le singularisent, qui l'extraient de toute durée où peut seule avoir lieu la répétition et non la reprise à l'identique de la première fois. Et ce alors même que la répétition apparaît comme fondatrice puisqu'elle est «la preuve que, même redite, une pensée ne se répète pas ou encore que la répétition fait seulement entrer ce qui se dit dans sa différence essentielle ${ }^{9}$ ». Différence essentielle que Derrida nomme " différance ", cette non-identité à soi qui est la condition même de la présence et de la répétition, liée à l'idéalité de la forme de la présence elle-même ${ }^{10}$. La différance est bien le fait d'être toujours déjà autre chose que soi-même, de se tenir ailleurs que dans sa propre présence. Les personnages de L'Attente l'oubli sont ainsi « détournés de leur présence pour être présents l'un à l'autre » $(A O, 72)$ : la vraie présence se donne dans la différence, dans la non-coïncidence avec soi. Le but et la chimère du récit, comme re-présentation, se tiennent là : accueillir la différence et saisir, dans son instantanéité essentielle, un dit premier sans l'altérer par la durée qu'impose pourtant tout récit. But et chimère puisque l'idéalité, autre "nom de la permanence du même et [de] la possibilité de sa répétition », «n'existe pas dans le monde ${ }^{11} »$.

6 C'est pourquoi, confronté à cette velléité nécessaire de saisir la dérobade du moment, c'est l'attente qui devient le viatique à même de figer l'évanescent. Notion capitale s'il en est dans l'univers fictionnel de Blanchot, l'attente est d'abord l'espace dilatoire où s'infiltrent la parole et la pensée entre les personnages. De fait, elle a partie liée, et ce intimement, avec la nécessité de la saisie temporelle, qu'elle soit verbale ou psychique. L'attente est le subside qui permet d'approcher l'instant, en l'étirant hors de lui-même. C'est pourquoi il semble bien que cette attente, au-delà d'une réalité en soi qui appartiendrait à la temporalité du monde, résulte avant tout du dire, de l'écrire et du penser. Elle s'inscrit dans la sphère du langage et de la subjectivité plus qu'elle n'existe en dehors des êtres et de leurs mots. Elle a partie liée avec la parole et le récit. Pour preuve, c'est bien le perpétuel dialogue relancé de L'Attente l'oubli qui est l'intercesseur privilégié de l'attente dans le réel. Ce sont les personnages qui attendent, et leurs mots qui la créent en dilatant le moment.

7 Néanmoins, ne pourrait-on penser que, suscitée par le discours et les personnages, l'attente soit dans le même temps livrée à une sorte de processus d'autonomisation? En effet, muée en entité propre, elle s'évide et se prive de contenu :

Dans l'attente, il ne pouvait questionner sur l'attente. Qu'attendait-il, pourquoi attendait-il, qu'est-ce qui est attendu dans l'attente? Le propre de l'attente est d'échapper à toutes les formes de question qu'elle rend possibles et dont elle s'exclut. $(\mathrm{AO}, 77)$

8 Sans but et contre toute détermination, l'attente arrête le devenir pour pérenniser l'instant. Sa fonction dans le récit est alors d'être la possibilité du récit et de son rapport à l'instant. Mais loin d'une mythification de l'attente, la notion se déleste de toute compromission avec une quelconque faculté de révélation : « nous sommes là pour garder le secret» $(A O, 104)$, affirment les personnages. L'inviolabilité du texte est l'écran 
réservant l'accès au sens qui s'apparente ainsi à l'illisible même. C'est dès lors le dit nondit qui se cherche sans se trouver dans l'attente: «ce qui est caché, cela s'ouvre à l'attente, non pour se découvrir, mais pour y rester caché » $(A O, 102)$. Par le secret, le dit s'essentialise. Mais les verrous et serrures s'accumulent. Car s'affiche ici l'une des difficultés majeures à laquelle s'affrontent autant les fictions que la réflexion théorique de Blanchot: avec un sens partout en voie de néantisation qui détournerait du phénoménal, tout renvoie finalement cette quête à son impossibilité alors même que la littérature, comme le monde, semblent attachés à une nécessaire condition phénoménale. C'est uniquement dans ce jeu de renvoi que le récit chez Blanchot laisse apparaître sa véritable nature. Rivé à un Neutre asymptotique et hors d'atteinte, le texte se voit contraint à saisir le phénoménal à la place, mais un phénoménal singulier qui ne peut s'admettre comme objectal. Car "dans l'attente, toutes choses sont retournées à l'état latent. » $(A O, 103)$ La latence dans l'attente, résorbées l'une dans l'autre sous les auspices de la quasi homophonie entre "l'attente» et "latent", est le véritable enjeu transcendant tout objet. Il ne faut donc pas céder aux Sirènes du mystère qui tentent de séduire le lecteur. L'objet du secret comme sa révélation sont nuls et non avenus. Le phénomène ne réside pas dans l'objet. Celui-ci est l'accessoire qui doit être tu puisque le seul ordre phénoménal qui importe est le processus de la quête de saisie du monde.

Signe syntaxique par excellence de cette capacité à dilater le moment et à inscrire l'attente: la multiplication des participes présents. Ceux-ci mettent en jeu un aspect sécant qui se généralise, effaçant ainsi les limites traditionnellement imparties au procès. Essaimés sous forme de chapelets, souvent dans des phrases nominales dont ils forment l'unique noyau verbal non actualisé, ils accompagnent la recherche de la suspension et de l'in posse :

Veillant sur l'insurveillé $(A O, 68,69)$

Voulant et ne voulant parler; ne le voulant pas et ne pouvant se dérober à la parole, alors parlant-ne parlant pas, dans un même mouvement que son interlocuteur avait le devoir de soutenir.

Parlant, ne le voulant pas ; le voulant, ne le pouvant pas. $(\mathrm{AO}, 71)$

Cherchant à endiguer le développement syntaxique normé, les participes présents éludent autant le sujet de l'action que son objet, puisqu'ils ne sont la plupart du temps reliés à aucun référent précisé, pouvant s'appliquer à l'un ou l'autre des personnages, aux deux, ou à tout être en général. Mode impersonnel par excellence, l'infinitif supprime de même autant l'actant que la situation du procès dans le temps :

Attendre, seulement attendre. $(\mathrm{AO}, 24)$

Accueillir l'oubli comme l'accord avec ce qui se cache, le don latent. (AO, 67)

11 Dans ce sillage, la généralisation des phrases nominales sous formes d'aphorismes vient déchirer la cohérence de la diégèse et empêcher de la constituer en durée. Ce phénomène de fragmentation du texte sous forme d'îlots est renforcé à l'attaque des paragraphes par la présence de signes typographiques qui les retranchent les uns des autres et accroissent ainsi les césures :

Dans l'attente, toute parole devenue lente et solitaire. $(A O, 40)$

Dans l'attente, le temps perdu. $(A O, 72)$

L'attente comblée par l'attente, comblée-déçue par l'attente. $(A O, 76)$

Blanchot semble alors soumettre son récit au phénomène de "discohérence» que Ricardou avait pu étudier dans le Nouveau Roman, cet assemblage de présences logiques irréductibles entre elles qui ouvre à une "cohérence contradictoire ${ }^{12}$ ». A l'échelle syntaxique ce phénomène est particulièrement prégnant. Une affirmation comme 
«Parlant, différant de parler» $(\mathrm{AO}, 84)$, propose par exemple une tension propre à la "discohérence " entre une action en cours et sa non réalisation. Ainsi les actions s'immobilisent, conjoignant l'instant et la durée, recherchant l'instant au cœur d'une durée surajoutée. Marquant des césures dans la chaîne syntaxique, les actions ne reconstituent en réalité pas un déroulement, soulignant chaque fois l'apparition d'un nouveau présent qui laisse entendre le vide qui le sépare du précédent et du suivant. Blanchot fait ainsi violence à la durée du récit en s'attaquant à sa syntaxe instituée.

La force de l'action est de la sorte très souvent contenue dans un verbe à l'état nu, sans amarre ni dans le sujet ni dans l'objet, d'autant que l'objet est fréquemment un « cela » ( $A O, 85)$, une puissance du Neutre qui, en soi, importe peu. C'est pourquoi le sabotage du récit s'accompagne d'un refus des cadres de la syntaxe et de la mise en œuvre d'une singulière grammaire narrative qui vise à obtenir les conditions propices à la mise en place de sa phénoménologie propre. Ce sont en effet les pouvoirs de l'intransitif et de l'inaccompli que les textes appellent de leurs vœux. Ainsi s'agit-il, bien plus que d'oublier quelqu'un ou quelque chose, d'amputer le verbe de son complément : « elle ne l'oubliait, elle oubliait » $(A O, 50)$. Les verbes demander, entendre, parler, penser, ou oublier, sont la plupart du temps intransitifs ou, s'ils s'attachent à un complément mentionné, ce dernier reste obscur, comme sans objet désigné.

L'instant se recherche ainsi paradoxalement dans ce qui le nie puisque «l'accompli demande son accomplissement » $(A O, 97)$. Il n'a nulle complétude dans son achèvement et doit renouer avec son propre procès, celui que lui imprime l'attente comme le récit. C'est pourquoi il semble bien que, chez Blanchot, plus qu'un accès direct au Neutre, on puisse parler d'un processus de neutralisation. Le Neutre, en soi accompli, appartient au domaine de l'inaccessible, et, dans les récits, sa présence ressortit plus au processus de l'absentement qu'à l'absence proprement dite.

\section{La mise en mots : une nécessité mortifère}

En creux de la plupart de ses textes, Blanchot laisse entendre, comme à mi-voix, que l'univers lacunaire et opaque du réel souscrit sans le savoir à l'ordre du livre. Ainsi le narrateur est-il souvent désigné, dans des mentions laconiques, comme celui qui écrit, sans être affilié explicitement à l'écrivain. Les premiers mots de L'Attente l'oubli montrent un monde comme par avance résorbé dans le dicible:

Ici, et sur cette phrase qui lui était peut-être destinée, il fut contraint de s'arrêter.

C'est presque en l'écoutant parler qu'il avait rédigé ces notes. Il entendait encore sa

voix en écrivant. Il les lui montra. Elle ne voulait pas lire. $(A O, 7)$

La matérialité de l'instant et du lieu est celle de la lettre, qu'elle soit écrite ou dite. Le texte répond aux pulsions d'une voix exogène à celui qui écrit, et se soumet à son ordre. L'entrée dans la fiction se veut ainsi entrée dans le monde du langage. Mais entrée paradoxale que le personnage féminin invite à refuser comme allant de soi. Le lecteur qu'incarne la jeune femme témoigne en effet d'une répulsion du texte soumis dès lors au soupçon de l'illisible comme du mensonge. C'est bien la différence entre l'écriture et la voix, sur laquelle Derrida avait attiré l'attention, qui se dit. La jeune femme refuse de lire la «trace» de ses propres paroles, elle refuse ce processus qui «fait descendre (...) le sens » mais qui élève " du même coup l'inscription ${ }^{13}$ ». En effet, l'écrit serait bien cette médiation qui fait passer le signifié par le signifiant, qui expose directement au monde, qui rompt cet état, propre à la voix, où le signifiant est si proche du signifié qu'il en 
devient « diaphane ${ }^{14}$ ». La jeune femme s'affole devant ce moment où au lieu d'« entendre parler, je (...) vois écrire ${ }^{15} »$. Le récit attire de fait l'attention du lecteur vigilant sur un point essentiel : le texte est un risque. Ce risque, la jeune femme le ressent, elle qui, à être ainsi mise en mots, contre son gré, $a$ « perdu le centre d'où rayonnaient les événements et qu'elle tenait si fermement jusqu'ici » (AO, 7-8). Le filtre des mots est bien ce qui empêche la présence immédiate à soi. Partant, le texte affiche sa nature blasphématoire puisqu'il s'en prend de toute évidence à l'épicentre même du monde, des hommes et de la narration : l'événement. Mais, à suivre cette voie là, il ne s'agit pas d'extrapoler cette qualité paradoxale de subversion à n'importe quel écrit. Le texte-filtre, crible, et frondeur, qui se définit en ce début de récit, n'est nullement le roman comme il s'entend traditionnellement mais bien le récit tel que Blanchot le conçoit. C'est donc avant tout le texte même de L'Attente l'oubli qui s'inscrit en abyme en ce début. Texte illisible, texte repoussant, texte anti-événementiel. C'est-à-dire le texte qui propose une phénoménologie du récit plus que du monde.

Et pourtant, ce grimoire magique et terrifiant que semble être le texte, ne saurait être tout puissant s' « il n'y a rien là qui puisse être exprimé par les mots les plus communs » ( $A O, 21)$. Partout, chez Blanchot, l'univers réel, précipité au cœur du dicible et du scriptible, se heurte à ses propres limites et faiblesses. C'est pourquoi, dès que le narrateur de L'Attente l'oubli essaye de décrire la chambre, dont la principale caractéristique est le «vide» $(A O, 14)$, «les mots dont il se sert ne recouvrent que le vide» $(A O, 15)$. Relégué au rang d'une tapisserie qui ne peut que couvrir d'une surface factice une lacune fuyante, le langage est condamné à une impuissance qui provient autant de sa nature que de celle de son objet. L'écrivant chez Blanchot, plus que l'écrivain, est ainsi celui qui dispose d'un "grand pouvoir dont il aurait pu se servir pour parvenir au cœur de cette vérité » que le monde exhibe à ses yeux $(A O, 15)$. Et pourtant, «de ce pouvoir, par une négligence extraordinaire, il refusait de rien faire ». Refus des charmes du mot ou charmes qui se refusent ? C'est dans cette tension que l'écriture se dit.

Croisant une réflexion sur l'être au monde et le récit, inextricablement liés, les textes de Blanchot proposent dès lors d'envisager l'être parlant dans ses rapports de proximité avec une instance narrative. En effet, toute mise en mots, apparentée à la démarche du narrateur, confine à une tentative de saisie d'un réel devenu le lieu d'une aporie. Puisque «le présent vivant jaillit de sa non-identité à soi, et de la possibilité de la trace rétentionnelle ", puisqu'il « est toujours déjà une trace ${ }^{16}$ ", le verbal serait le seul ordre à même d'approcher le monde, mais sans pour autant pouvoir le rendre dans sa condition évanescente d'apparaître instantané. De la sorte, Blanchot exhibe tous les simulacres et toutes les faillites du récit, autant de lignes de fissures qui viennent déchirer de l'intérieur la quête d'une saisie du monde. Aussi, le narrateur est-il celui qui a pour difficile tâche de nommer le monde. C'est bien le rôle dans lequel ne se voit pas le «je » du Dernier homme puisque "le nom même nous sépare ${ }^{17}$ ». En obligeant le personnage qu'est le dernier homme "à se reconnaître sous un tel nom », le narrateur lui tend un " piège ». Toute tentative de saisir l'être, et surtout textuelle ou verbale, l'enferme. Là se tient donc le paradoxe du récit chez Blanchot : le mot exprime et pourtant «fait écran à l'expression » $(A O, 75)$.

19 Mais les difficultés se redoublent dès lors que l'on envisage que ce tropisme pour la mise en mots n'est pas uniquement la résultante d'un besoin de saisie du monde. Car, chez Blanchot, c'est l'autre qui instille dans le moi la nécessité de le dire. Le personnage parlant est toujours de la sorte aussi un personnage parlé : «Elle parle, parlée plutôt que parlant, 
comme si sa propre parole la traversait vivante et la transformait douloureusement en l'espace d'une autre parole» $(A O, 112)$. Ce sont bien, au départ de L'Attente l'oubli, les phrases issues de la voix féminine, qui parlent dans le narrateur $(A O, 7)$. Dans la même perspective, le narrateur qui prend la parole face au «dernier homme» est comme possédé par celui dont il parle: «telle phrase que je lis, que j'écris, s'écarte pour faire place à la sienne » $(\mathrm{DH}, 10)$. Evincé par son personnage au moment où il commet l'acte suprême qui confine à l'échec, écrire, le narrateur, et avec lui tous les narrateurs chez Blanchot, est pris dans les rets d'une parole maléfice qui fait intrusion en lui. Le dernier homme est à cet égard exemplaire en ce qu'il dépose chez le narrateur " des repères » (DH , 47) verbaux, sorte de concrétions exogènes. Ces dépôts de l'être, qui se réalisent dans la parole, sont soumis à une forme de stagnation délétère, puisque toute nomination est un " arrêt de mort » pour l'être ou pour la chose :

Paroles immobiles que je sens à présent, à cause de cette immobilité qui m'avertit de quelque chose, et les rend pesantes, légères ? Trop légères pour celui qui, au lieu de les laisser venir à elles-mêmes, ne peut que les fixer, sans l'espace vivant où elles s'animeraient. $(\mathrm{DH}, 48)$

L'être blanchotien, ce narrateur contre son gré, est absolument conscient de son impuissance à se défaire du contrôle qu'il exerce naturellement sur les mots, pour les laisser vibrer dans un espace lui échappant et échappant à l'ordre du langage. De ce point de vue, le récit et l'attente sont frères dans la durée qui les constitue :

Attendre semble signifier pour elle la remise d'elle-même à une histoire qu'elle lui ferait une obligation de mener à bien et qui doit avoir pour conséquence sa marche progressive vers un but. L'attention devrait être comme exercée par ce récit de manière à l'arracher lentement à la distraction initiale sans laquelle pourtant il sent bien que l'attention deviendrait un acte stérile. $(A O, 17)$

Synonyme d'une reddition de l'être à la durée de la narration, le récit instaure un progrès et un déroulement qui nient l'instant, même si l'attente qu'il génère est le seul recours pour parvenir conjointement à l'attention extrême à l'instant et à la distraction absolue à soi, conditions nécessaires à l'accès à la présence du moment et de l'être. Le récit est ainsi une nécessité autant qu'une entrave. C'est pourquoi le seul récit à même de garantir l'évidement du contenu est celui qui opte pour « un refus énergique de laisser l'histoire débuter " $(A O, 18)$, qui conteste l'événement ${ }^{18}$. Amputé d'une entrée dans le système structuré du narratif, le récit peut ainsi se concevoir comme une suspension absolue et un ressassement perpétuel. Lieu de la « discohérence » narrative comme syntaxique, le récit répète les mêmes actions des personnages, les ressasse, et les rend problématiques en les niant au fur et à mesure qu'elles se réalisent. Une absence de cohérence factuelle s'observe dans l'enchaînement des actes des personnages. De même, leurs pensées se contredisent régulièrement. Les textes de Blanchot perdent alors les codes de l'intrigue, les règles tyranniques de la diégèse, et refusent de s'assigner aux cadres inéluctables de l'incipit comme du dénouement. C'est le surplace qui préside à leur mise en œuvre : « Le sens de toute cette histoire était celui d'une longue phrase qui ne pouvait être morcelée, qui ne trouverait de sens qu'à la fin et qui, à la fin, ne le trouverait que comme un souffle de vie, le mouvement immobile de tout l'ensemble » (20). Une circularité obsessionnelle qui gravite autour du point aveugle qu'est l'instant dérobé. C'est pourquoi la diégèse se doit d'être liquidée. L'impératif est clair: "il ne doit plus rester grand-chose de l'histoire » $(A O, 115)$. 


\section{L'oubli de l'instant et l'instant de l'oubli}

22 avec la naissance du verbe. Mais, devant les déchirements inhérents à cette posture, c'est une deuxième force exceptionnelle dans l'économie de l'être redéfini à l'aune du Neutre qui est convoquée: l'oubli. En tension, ces deux pôles s'influencent et modifient respectivement leurs contours et leurs possibilités. Apparemment placées en contradiction, les notions se nouent pourtant résolument, dès le syntagme-titre qui les amalgame et où le tiret, puisqu'il indiquerait peut-être plus une césure qu'un lien, s'est absenté : «L'attente l'oubli ». La préservation des deux déterminants définis, qui exhibent l'autonomie de chaque entité, ne doit pas faire oublier leur complémentarité. Car une visible circularité entretient leurs rapports. C'est ainsi en empruntant la logique du chiasme que Blanchot les met en communication intime : «L'oubli, l'attente. L'attente qui rassemble, disperse ; l'oubli qui disperse, rassemble. L'attente, l'oubli. » $(A O, 49)$ Plus qu'à observer une virtuosité gratuite, Blanchot invite son lecteur à pénétrer dans le domaine obscur où l'esprit est forcé de penser les logiques paradoxales et circulaires. Par ce règlement de la pensée sur la " différance ", " les concepts deviennent des non-concepts, ils sont impensables, ils deviennent des intenables ${ }^{19}$." La cohérence textuelle traditionnelle ne peut s'établir à cause de cette conjonction d'éléments contradictoires et pourtant indissociables. Blanchot offre ainsi une vision du monde sans unité, irréductible aux catégories de l'unité, de la pluralité, de la causalité ou de la substance. C'est autant notre réel que notre saisie du réel par le récit qui se redéfinissent.

Le jeu vertigineux qui met en tension et en communication l'attente et l'oubli, répété en plusieurs endroits du texte, est finalement essentiel. En effet, alors que l'attente est la puissance même qui fournit à l'être sa tension nécessaire vers l'instant, l'oubli semble lui ravir cette disponibilité. Néanmoins, il s'agit de comprendre que l'attente et l'attention ne peuvent aussi se passer d'une "distraction", condition sine qua non de la non-identité à soi et de l'évidement d'un concret dont le débordement de substance empêche de penser l'essence vide. De fait, c'est bien l'oubli en soi qui fonctionne ici à plein régime, indice même de la distraction absolue qui est réclamée devant l'univers surchargé de contenu et de matière qui est le nôtre. Si «l'être est encore un nom pour l'oubli » $(A O, 52)$, il s'agit toujours d'« user la présence" $(A O, 79)$ afin de voir à travers, "comme si l'effacement était le lieu dernier de toute rencontre. » $(A O, 52)$ Effacer une présence pour ouvrir à la non-coïncidence et, enfin, par le jeu de la différence, faire jaillir la présence du présent. Ainsi, répondant à la pulsion fondatrice qui polarise le personnage vers l'attente, le désir obsessionnel de l'oubli s'empare de la plupart des personnages de Blanchot puisque seule "l'indifférence précis[e] la présence» $(A O, 78)^{20}$. Happés par une apostasie et une dissolution continuées de soi, où l'anonymat oriente l'écrit vers les puissances du Neutre, les personnages heurtent l'utopie rassurante d'une consistance du sujet.

Placée sous le signe de l'oubli, la phénoménologie du récit affronte alors une difficulté supplémentaire : celle de la trace écrite attachée tragiquement à la préservation dans le temps, celle d'une écriture qui "n'est une trace que si en elle la présence est irrémédiablement dérobée (...) et si elle se constitue comme la possibilité d'un effacement absolu $^{21}$ ». La question essentielle est ainsi : comment proposer un récit oublieux plus qu'un récit de l'oubli? Car le problème n'est pas tant de peindre des personnages amnésiques. Peu importe en effet les pathologies de la mémoire en tant que telles. Ce qui

Cahiers de Narratologie, 22 | 2012 
importe au contraire est que le récit n'aille pas contre la marche délibérée de la pensée de l'instant qui a besoin d'être soutenue par l'oubli comme puissance d'absentement. Se joue donc la recherche d'un récit sans fixation et sans mémoire, rendu uniquement à son " avoir-lieu » et à son "événement ${ }^{22}$ ». Cette exigence, qui tient dans l'ensemble des textes, explique les nombreux refus que l'œuvre porte : celui d'une intertextualité visible, celui de la référence ${ }^{23}$. De la sorte, la quête du texte passe par la recherche d'une parole neuve et comme lavée, jusqu'à être usée et délavée, sans amarres dans le déjà-dit. C'est pourquoi, alors que le texte est contraint à la fixation délétère, les récits de Blanchot partent à la recherche des «mots faits pour l'oubli » $(A O, 58)$. Des mots non pas faits pour être oubliés, mais pour porter l'oubli. L'oubli doit « demeure[r] en une parole. » $(A O, 52)$. De la sorte, le langage chez Blanchot se veut sans épaisseur et sans alibi. La jeune femme de L'Attente l'oubli ne sait pas "relier les mots à la richesse d'un langage antérieur » ( $A O$, 19). Ceux-ci sont « sans histoire, sans lien avec le passé de tous, sans rapport même avec sa vie à elle, ni avec la vie de personne. » $(A O, 19-20)$ La règle du langage s'appuie sur celle de l'oubli : «oubliant un mot, oubliant en ce mot tous les mots » $(A O, 67)$. Se délestant de lui-même, le langage se love dans l'oubli comme il devient un réceptacle à même de l'accueillir :

Il était étrange que l'oubli pût s'en remettre ainsi à la parole et la parole accueillir l'oubli, comme s'il y avait un rapport entre le détour de la parole et le détour de

l'oubli.

Ecrivant dans le sens de l'oubli. $(\mathrm{AO}, 68)$

Usée elle-même par l'oubli, la parole est le viatique qui laisse entendre la vibration nue de cet oubli. L'oubli phagocyte ainsi son objet et efface ses traces : «L'oubli est rapport avec ce qui s'oublie, rapport qui, rendant secret cela avec quoi il y a rapport, détient le pouvoir et le sens du secret » $(A O, 67)$. Mais, éliminant l'événement, l'oubli inscrit aussi la trace de son absentement. C'est pourquoi l'événement chez Blanchot ne connaît que deux modalités en miroir capables d'abriter sa présence, l'interrogation et la négation : «"Estce que cela arrive ?" - “Non, cela n'arrive pas." » $(A O, 114)$.

Le risque de stase du récit se voit ainsi hypothéqué par le redoublement de ce qu'on pourrait appeler une phénoménologie de l'instant oublié par une phénoménologie de l'instant de l'oubli :

L'événement qu'ils oublient : événement de l'oubli. Et ainsi, d'autant plus présent qu'oublié. Donnant l'oubli et se donnant oublié, mais n'étant pas oublié. Présence d'oubli et en l'oubli. Pouvoir d'oublier sans fin en l'événement qui s'oublie. (AO, 110-111)

Il y a là comme un jeu d'éloignement à l'infini, déplaçant le contenu vers la forme puis la forme vers une autre forme. En effet, tout porte à croire que, si l'instant est oublié, menaçant toute saisie de cet instant, demeure une possibilité dernière : la tentative de saisie de l'instant où l'instant a été oublié. Dans l'absentement radical qui se joue ici, l'événement s'efface devant l'événement de l'événement, redéfinissant ainsi définitivement la nature du récit.

\section{L'événement du récit}

Si le monde réel se déprend des sujétions de l'objectal, il est inévitable que ce mouvement de déport entraîne dans son sillage l'ensemble du récit. Ces nouvelles règles édictées, le récit, comme espace même où s'origine la pensée et la parole, se rêve véritable apparaître 
et véritable événement. Pensé dans l'orbe de la forme, et non dans celui du contenu, le récit, en s'évidant, se réinvente. C'est au sein de ce nouveau paradigme qu'il convient d'envisager la pratique du récit chez Blanchot, radicalement distante du factuel, et s'apparentant de fait au dire d'un dit. Puisque appréhender l'instant comme le dit relèvent d'une velléité impossible, la seule approche est celle du dire et du récit qui est la trace du dit premier perdu, non son inscription mais celle de sa quête, à la manière dont l'attente est ce qui reste de l'instant. Puisque l'instant est indicible une deuxième fois et ininscriptible par la narration, reste sa recherche qui appartient seule au domaine du scriptible. C'est pourquoi le récit, dans sa quête d'un instant dérobé, se pense selon une démarche métonymique de la contiguïté : "Quand il se retourne vers le temps où il lui a fait signe, il sent bien qu'il lui fait signe en se retournant » $(A O, 111)$. Le signe premier est toujours opaque et inaccessible : sa recherche, son inscription, c'est-à-dire le récit, font alors signe à sa place en se tournant vers lui. Ils en sont la véritable trace. «Le langage poétique, dit à juste titre Lévinas au sujet de Blanchot, fait signe sans que le signe soit porteur d'une signification en se dessaisissant de la signification ${ }^{24}$ ». Ce n'est donc pas l'instant du signe en soi qui signifie désormais mais bien l'instant où l'on pense à, ou parle de l'instant du signe : l'instant qu'est finalement le récit. Comme l'affirme Derrida, «ce qui se récite ici, cela aura été cette non-présentation de l'événement, sa présence sans présence ${ }^{25}$ ». La place fondamentale du dialogue en témoigne. En effet, l'acte du dialogue est essentiellement non son énoncé mais son énonciation, analogon d'un rapport singulier entre les êtres, puisque expurgé de l'encombrante nécessité de la fonction référentielle. Après l'un des nombreux dialogues de L'Attente l'oubli, c'est bien l'acte même du dialogue qui emporte la signification principale: «Il sentait qu'en effet cette pensée ne leur était pas commune, mais qu'ils ne seraient en commun que dans cette pensée. » ( $A O, 62)$ L'acte en soi, le dire, la pensée, la parole, le récit, transcende toujours un quelconque contenu. Débarrassé des scories de la contingence que sont le passé, la psychologie, la parole quotidienne, toutes ces compromissions de l'apparaitre et de l'événement avec le tangible, le récit se livre dans une pure nudité. C'est pourquoi «la question que l'attente porte en elle : elle la porte, sans se confondre avec elle.» $(A O, 73)$ De la sorte, s'il n'existe jamais de « réponse qui épuiserait la question » $(A O, 74)$, c'est bien que la signification réside non dans le contenu mais bien dans l'acte d'interroger. De ce fait, les mots semblent comme par nature appeler ce phénomène, puisqu'ils sont en quelque sorte redevables de cette " passion d'apparaître » qui tenaille le personnage ( $A O$, 97). Ils n'importent donc pas en eux-mêmes mais seulement par leur faculté d'apparaître et par leur statut d'événement, qui objectivent leur présence absente: "Dans chaque mot, non pas les mots, mais l'espace qu'apparaissant, disparaissant, ils désignent comme l'espace mouvant de leur apparition et de leur disparition.» (AO, 101-102). Le récit se brise selon le mouvement de flux et de reflux des apparitions et disparitions, jusqu'à celui des mots eux-mêmes, dans ce que Françoise Collin nomme un « apparaître-disparaître ${ }^{26}$ ".

Cette conception du récit décide dès lors d'un état de fait : la plupart du temps, tout a déjà été dit, fait ou pensé. L'événement est par avance réalisé et n'a pas droit de cité au sein de la narration, à la manière dont le malheur, comme le notait Georges Poulet, n'apparait « jamais comme un événement actuel, mais comme la seule explication d'un état qui est lui-même par-delà le malheur ${ }^{27}$ ». La place de l'événement est ainsi toujours extrêmement restreinte, à peine suggérée lorsque celui-ci est présent, toujours décalée. «Supposez que ce que vous me demandez, vous me le demandiez parce que je l'aurais déjà fait » $(A O, 62)$, propose la jeune femme. «Pourriez-vous me demander une telle chose, si vous ne l'aviez 
déjà apprise et pénétrée par son accomplissement en vous-même ?» L'allusion à la chose, renvoyée dans le réalisé, n'est jamais autrement spécifiée. Le récit se construit ainsi sur une tension entre l'imperfectif de l'attente, tendue vers le "moment voulu», et le perfectif de l'événement expulsé dans les coulisses hors-scène du texte. Relégué dans une antériorité ni située ni situable, effaçant tout référent possible, l'événement s'engouffre dans le processus d'absentement et de neutralisation: il invite à dépasser tous les contenus, que ce soit le sien, celui de l'instant, le dit ou l'objet. En somme, à la catégorie de l'accompli, s'attache le contenu, alors que dans la catégorie de l'inaccompli se déploie l'acte. De cet anathème à l'encontre du factuel ne naît pourtant aucun sentiment tragique qui, par nature, se fonde sur l'inéluctable de l'accompli par avance. Le récit se place et place ses personnages au cœur de «ce cercle de l'attrait où l'on ne commence à parler que parce que tout a déjà été dit » $(A O, 53)$. Epicentre fascinant qui organise autour de lui la gravitation de l'ensemble des éléments du texte et qui fonde la singularité de l'événement du récit. Le récit est bien le seul lieu de la présence, là où peut se tenir la répétition et la re-présentation. Il est avant tout ré-citation.

Et, presque au terme de L'Attente l'oubli, les personnages semblent avoir usés jusqu'au bout leur être et leur attente :

« Nous n'attendons plus, nous n'attendrons plus jamais. - « C'est que nous n'avons jamais vraiment attendus. » - " Tout a donc été inutile ? Tant d'efforts dissipés, tant de moments arrêtés.» - «Nous fûmes patients et immobiles.» - «Il n'est pas nécessaire, maintenant, que nous parlions. Restons tranquillement à nous entendre. » $(A O, 102)$

31 Pourquoi ce constat d'échec alors même que l'attente a été l'objet de la plus vive opiniâtreté ? Parce que, juste avant, les personnages se sont accordés sur le fait que la répétition n'était pas possible. L'attente paraît donc vaine puisqu'elle ne fera pas revivre l'instant de la parole. Néanmoins, elle a permis le récit avec les paroles dont il s'est chargé. Même si elle n'a pu ni susciter ni ressusciter l'instant premier du mot, celui qui échappe toujours, elle a donné naissance à une forme de répétition, par la ré-citation, par la voix, celle des personnages et celle du récit. L'Attente l'oubli semble ainsi appeler cette " voix phénoménologique » qui, selon Derrida «serait cette chair spirituelle qui continue de parler et d'être présente - de s'entrendre - en l'absence du monde ${ }^{28}$.» Retranchés du monde comme de l'énoncé, l'énonciation et le récit garantissent seuls la présence. Il s'agit bien pour Blanchot de concevoir un récit placé sous le règne de ce médium qui est capable d'exprimer et de répéter, qui "n'entame pas la présence ${ }^{29}$ » et où le corps phénoménologique de l'énoncé semble s'effacer dans son énonciation. Même si cette voix essentielle, cette voix phénoménologique, ce dit à l'état nu, ne se réalise pas pleinement, même si la parole reste compromise avec les simulacres du récit, de la fiction et de sa durée, avec le dehors qu'impose l'écrit, avec cette «mort dans le corps d'un signifiant abandonné au monde ${ }^{30} »$, cette voix en forme de pis-aller est la seule chose qui puisse témoigner de l'inaccessible et du silence qui entoure le dit essentiel pris dans l'instant, ce dit toujours non-dit et hors d'atteinte. En effet, le texte pose le lien essentiel mais ténu qui relie l'attente au récit :

L'attente : attiré par l'attente dans cet intervalle entre voir et dire qu'il ne supporte qu'à la faveur de l'histoire et où celle-ci se joue en déployant son jeu, mais aussitôt - et peut-être dès l'abord - rejeté par la vérité du jeu de l'histoire vers l'attente qui les retient l'un et l'autre, comme à l'écart de la présence. $(A O, 103)$

L'attente est « la voie conduisant de l'événement qu'elle attend à l'histoire où elle attend, l'un et l'autre maintenus ensemble par l'oubli » $(A O, 104)$. Le récit s'affiche bien comme le 
passage de l'instant attendu au lieu de l'attente: son événement est donc la seule voie d'accès à l'instant mais en même temps l'échec de sa saisie, déplacé depuis le contenu vers le contenant.

L'Attente l'oubli est donc bien le texte où s'affiche avec le plus de force la volonté de redéfinir le cadre du récit, mais dont on trouve des traces dans presque toutes les fictions de Blanchot $^{31}$. Plus qu'un récit comme pensée de l'événement de la vie, il s'agit de comprendre l'événement du récit comme pensée de l'événement de la vie. Les textes interrogent ainsi le récit potentiel que sont l'être, l'événement et le monde. Convoquant l'attente et l'oubli, le récit part en quête d'un instant et d'un dit premiers inaccessibles. Devant l'évidement des contenus et des substances, c'est donc une phénoménologie nouvelle qui s'invente, une phénoménologie du récit qui oriente son lecteur vers une zone trouble et opaque : celle où le contenu s'efface devant la forme. Ainsi c'est bien l'événement du récit, en quête de l'événement de l'oubli, de l'attente, de l'instant ou de la parole, qui est seul à même d'opérer cette quête. S'il semble bien que la pensée philosophique de Blanchot soit tenue par l'exigence d'affronter l'impensable du Neutre et du sens absent, ses fictions essayent elles aussi de réaliser ce désir. L'Attente l'oubli est de la sorte l'expression même de la recherche de la voix phénoménologique, cette voix du récit qui, retranchée du monde, du sens et de l'énoncé, ferait vibrer la présence. Sans être la réalisation pleine de cette voix, L'Attente l'oubli s'affiche comme une tentative singulière de cerner les raccords et les discords entre le récit et le monde, autant de liens à l'origine de la fascination et de la nécessité qu'il a toujours entretenues chez l'homme.

\section{ANNEXES}

\section{Maxime Decout}

Principaux travaux :

"Albert Cohen, un solitaire dans la littérature française ", Les Temps modernes, n 656, novembre-décembre, 2009, p. 64-82.

«Le «parlécrit» chez Albert Cohen: d'une authentique version à une perversion du monologue intérieur ", Poétique, n 159, 2009, p. 311-324.

"Georges Perec : la judéité de l'autre », Roman 20-50, n 49, juin 2010, p. 123-134.

"Waiting for the Barbarians de Coetzee : réécrire la mort du Christ, refuser la croix », Actes du colloque L'Intertextualité dans le roman contemporain de langue anglaise, Jocelyn Dupont et Emilie Walezack (dir), Presses Universitaires de Perpignan, collection «Etudes », 2010, p. 93-107.

« «Le Juif et les romanciers français»: De Solal aux Rosenfeld, une plongée dans la fabrique du personnage juif chez Albert Cohen », Cahiers Albert Cohen, n 19, 2009, p. 51-74.

"L'animal dans le social: immobilisme d'une société ou impossible réforme de l'homme? », Cahiers Albert Cohen, $n^{\circ} 18,2008$, p. 99-116. 


\section{A paraître :}

Direction du numéro spécial d'Europe consacré à Georges Perec, 2011.

« Belle du Seigneur, une genèse contrariée », Genesis, n 32, mars 2011.

« Modiano : la voix palimpseste sur la place de l'étoile », Littérature, 2011.

\section{NOTES}

1. Voir entre autres Jacques Derrida, De la grammatologie, Paris, Minuit, "Critique », 1967 ; Georges Bataille, L'Expérience intérieure, Paris, Gallimard, « Tel », 1980 [1943].

2. Jacques Derrida, Parages, Paris, Galilée, 1986-2003, p. 121.

3. Voir à cet égard les réflexions de Blanchot dans L'Espace littéraire, Paris, Gallimard, «Folio essais », 1988 [1955], p. 213-224 ; L'Entretien infini, Paris, Gallimard, 1969, p. 421-636.

4. Marlène Zarader, L'Etre et le Neutre. A partir de Maurice Blanchot, Lagrasse, Verdier, "Philia ", 2001 , p. 25.

5. Ibid., p. 42.

6. Ibid.

7. Henri Bergson, Essai sur les données immédiates de la conscience, dans CEuvres, Paris, PUF, 1959, p. 87.

8. Maurice Blanchot, L'Attente l'oubli (AO), Paris, Gallimard, «L'imaginaire », 2000 [1962], p. 100-101.

9. Maurice Blanchot, L'Entretien infini, op. cit., p. 501.

10. Jacques Derrida, La Voix et le Phénomène, Paris, PUF, « Quadrige », 1998 [1967], p. 75-77.

11. Ibid., p. 58.

12. Jean Ricardou, Nouveaux problèmes du roman, Paris, Seuil, « Poétique », 1978, p. 231.

13. Jacques Derrida, L’Ecriture et la Différence, Paris, Seuil, « Tel Quel », 1967, p. 21.

14. Jacques Derrida, La Voix et le Phénomène, op. cit., p. 90.

15. Ibid., p. 90.

16. Ibid., p. 95.

17. Maurice Blanchot, Le Dernier Homme (DH), Paris, Gallimard, «L'imaginaire », 1992 [1957], p. 46.

18. Voir à cet égard la fin des deux parties qui constituent L'Arrêt de mort, Paris, Gallimard, «L'imaginaire », 1977 [1948], p. 53, 126-127.

19. Jacques Derrida, L'Ecriture et la Différence, op. cit., p. 393.

20. Voir à cet égard, entre autres, les personnages de Thomas l'obscur.

21. Jacques Derrida, L'Ecriture et la Différence, op. cit., p. 391.

22. Jacques Derrida, Parages, op. cit., p. 121.

23. Voir Christophe Bident, «Le secret Blanchot ", Poétique, $n^{\circ}$ 99, septembre 1999, p. 301-320.

24. Emmanuel Lévinas, «La servante et son maître. A propos de "L'attente l'oubli", Critique, n 229,1966, p. 520.

25. Jacques Derrida, Parages, op. cit., p. 176.

26. Françoise Collin, Maurice Blanchot et la question de l'écriture, Paris, Gallimard, «Tel », 1986 [1971], p. 77.

27. Georges Poulet, « Maurice Blanchot critique et romancier », Critique, n²29, 1966, p. 496-497.

28. Jacques Derrida, La Voix et le Phénomène, op. cit., p. 15.

29. Ibid., p. 85.

30. Ibid., p. 87. 
31. Assurément, seul Le Très-Haut échappe véritablement à cette définition du récit puisqu'il conserve une diégèse structurée de façon beaucoup plus traditionnelle.

\section{RÉSUMÉS}

Les récits de Maurice Blanchot, et tout particulièrement L'Attente l'oubli, dérangent les codes traditionnels du récit en l'évidant de ses personnages comme de son histoire. C'est pourquoi ils semblent proposer une nouvelle poétique du récit où le centre de gravité du texte se déporte peu à peu vers l'événement qu'est en lui-même le récit. Pour ce faire, ils tentent de saisir l'instant premier d'un dit essentiel en convoquant les forces de l'attente et de l'oubli, associées dans une perception renouvelée d'un réel soumis au Neutre et à l'absence de sens. Les contenus s'évident peu à peu pour laisser résonner dans leur nudité les formes et apparences qui les portent: l'énonciation, le dire, l'acte de penser, d'oublier ou d'attendre sont les seuls événements que le récit peut prendre en charge devant un énoncé, un dit, une pensée, un oubli ou une attente vidés de toute substance.

\section{INDEX}

Index géographique : France

Index chronologique : XXe siècle

Mots-clés : attente, énonciation, événement, Maurice Blanchot, oubli, phénoménologie, récit

\section{AUTEUR}

\section{MAXIME DECOUT}

Université Lille 3 Charles de Gaulles, ALITHILA. Agrégé de Lettres Modernes, titulaire d'une thèse sur la judéité dans l'œuvre d'Albert Cohen, à paraître chez Classiques-Garnier en 2011, et professeur de khâgne. 